\title{
Perfil do acadêmico de Enfermagem e o seu conhecimento sobre infecção do trato
}

\section{urinário: estudo descritivo}

\author{
Profile of Nursing academic and knowledge about urinary tract infection: descriptive study \\ Perfil do acadêmico de Enfermagem e o seu conhecimento sobre infecção do trato urinário
}

Recebido: 11/03/2021 | Revisado: 17/03/2021 | Aceito: 18/03/2021 | Publicado: 27/03/2021

\author{
Raphael Monteiro de Oliveira \\ ORCID: https://orcid.org/0000-0002-4321-2316 \\ Marinha do Brasil, Brasil \\ E-mail: raphael.universont@gmail.com \\ Luiza Francymara de Paula Miñan \\ ORCID: https://orcid.org/0000-0002-9789-9838 \\ Universidade Federal Fluminense, Brasil \\ E-mail: luizafrancymara@gmail.com \\ Rafael Pires Silva \\ ORCID: https://orcid.org/0000-0002-9786-3229 \\ Marinha do Brasil, Brasil \\ E-mail: raphael.universont@gmail.com \\ Bruna Maiara Ferreira Barreto Pires \\ ORCID: https://orcid.org/0000-0002-5584-8194 \\ Universidade Federal Fluminense, Brasil \\ E-mail: bruna_barreto@id.uff.br
}

\begin{abstract}
Resumo
Objetivo: Descrever o perfil do acadêmico de enfermagem de uma instituição privada e destacar qual o conhecimento do mesmo sobre Cateter Vesical de Demora. Metodologia: estudo descritivo, de abordagem quantitativa, realizado no período do segundo semestre de 2017 que visou à coleta de dados, de acadêmicos de enfermagem de todos os períodos do turno noturno através de um questionário estruturado e fechado. A pesquisa foi realizada em instituição de ensino superior de nível privada em Niterói, Rio de Janeiro, Brasil. Resultados: O perfil do acadêmico de enfermagem que ingressa em uma universidade privada, trata-se de um público de adultos jovens que já estão inseridos no mercado de trabalho e buscam a universidade, motivados pela ascensão da carreira, bem como melhoria de salários. O conhecimento dos acadêmicos torna-se homogêneo quando comparado no geral, mas quando separado dos profissionais de saúde, estes possuem um número de acertos maior, sugerindo que a atividade profissional pode ser um facilitador para assimilação do conteúdo. Conclusão: Conclui-se que o perfil sociodemográfico dos alunos de enfermagem não interfere no processo de aprendizagem e assimilação do conteúdo. Diante desses resultados, sugere outras pesquisas relacionadas aos dificultadores do processo de aprendizagem e o conhecimento do aluno sobre Infecção do Trato Urinário, bem como uma comparação do perfil acadêmico e o conhecimento do mesmo a cerca do tema em universidades públicas como comparativo e objetivo de melhoria na qualidade de educação e formação dos profissionais de enfermagem.
\end{abstract}

Palavras-chave: Ensino; Enfermagem; Infecções.

\begin{abstract}
Objective: To describe the profile of nursing students at a private institution and to highlight their knowledge about the Bladder Catheter. Methodology: a descriptive study, with a quantitative approach, carried out in the period of the second semester of 2017, aimed at data collection, of nursing students from all periods of the night shift through a structured and closed questionnaire. The research was carried out in a private higher education institution in Niterói, Rio de Janeiro, Brazil. Results: The profile of the nursing student who enters a private university, is an audience of young adults who are already inserted in the labor market and seek university, motivated by the rise of their careers, as well as improved salaries. The knowledge of academics becomes homogeneous when compared in general, but when separated from health professionals, they have a greater number of correct answers, suggesting that professional activity can be a facilitator for assimilation of content. Conclusion: It is concluded that the sociodemographic profile of nursing students does not interfere in the process of learning and assimilation of content. In view of these results, it suggests other research related to the hindering of the learning process and the student's knowledge about Urinary Tract Infection, as well as a comparison of the academic profile and the knowledge of the same about the theme in public universities as a comparative and improvement objective in the quality of education and training of nursing professionals.
\end{abstract}

Keywords: Teaching; Nursing; Infections. 


\begin{abstract}
Resumen
Objetivo: Describir el perfil de los estudiantes de enfermería de una institución privada y resaltar sus conocimientos sobre el Catéter de Vejiga. Metodologia: estudio descriptivo, con enfoque cuantitativo, realizado en el período del segundo semestre de 2017, dirigido a la recolección de datos, de estudiantes de enfermería de todos los períodos del turno de noche a través de un cuestionario estructurado y cerrado. La investigación se llevó a cabo en una institución privada de educación superior en Niterói, Río de Janeiro, Brasil. Resultados: El perfil del estudiante de enfermería que ingresa a una universidad privada, es una audiencia de adultos jóvenes que ya están insertos en el mercado laboral y buscan la universidad, motivados por el ascenso de sus carreras, así como la mejora de los salarios. El conocimiento de los académicos se vuelve homogéneo cuando se compara en general, pero cuando se separa de los profesionales de la salud, tienen un mayor número de respuestas correctas, sugiriendo que la actividad profesional puede ser un facilitador para la asimilación de contenidos. Conclusión: Se concluye que el perfil sociodemográfico de los estudiantes de enfermería no interfiere en el proceso de aprendizaje y asimilación de contenidos. A la vista de estos resultados, sugiere otras investigaciones relacionadas con la obstaculización del proceso de aprendizaje y el conocimiento del estudiante sobre Infección del Tracto Urinario, así como una comparación del perfil académico y el conocimiento del mismo sobre el tema en las universidades públicas como Objetivo comparativo y de mejora en la calidad de la educación y formación de profesionales de enfermeira.
\end{abstract}

Palabras clave: Enseñanza; Enfermería; Infecciones.

\title{
1. Introdução
}

A enfermagem desde o inicio de sua história é responsável pela execução dos procedimentos técnicos na atenção a saúde, principalmente ações de promoção, tratamento e reabilitação, voltadas a pacientes clínicos agudos ou crônicos (Brasil, 2013)

O profissional de enfermagem em sua atuação presta constante cuidado ao paciente em todo o seu período de internação e até mesmo no cuidado domiciliar. Muitas vezes cabe também a esse profissional o treinamento de sua equipe quanto à assistência e a adequada conduta a determinados procedimentos sendo grande atuante nas condutas de prevenção de infecção hospitalar.

O Cateterismo do trato urinário é um procedimento constante em grande parte da rotina hospitalar, e consiste na introdução de um cateter pela uretra até a bexiga com o objetivo de drenagem de urina, podendo este, ser utilizado a curto e longo prazo dependendo da indicação clinica para cada cliente.

O Conselho Federal de Enfermagem normatiza com a resolução de No450 em dezembro do ano de 2013, que a Inserção de sondagem vesical (SV), por tratar-se de um procedimento invasivo do qual requer cuidados de enfermagem de maior complexidade, conhecimento técnico, cientifico e capacidade de tomada de decisões rápidas, torna-se privativa do enfermeiro devendo o mesmo imprimir rigor técnico-cientifico na pratica e não podendo delegar tal conduta aos técnicos de enfermagem, considerando que se trata de um procedimento de alta complexidade.

O cateter vesical de demora (CVD) ou sonda vesical de demora (SVD) é a drenagem de urina por um tubo inserido pela uretra até a bexiga que permanece por tempo indeterminado, estando conectado há um sistema coletor fechado. E dentro das infecções relacionadas à assistência da saúde (IRAS), as Infecções do trato urinário (ITUs) são os tipos mais comuns, reportando mais de $30 \%$ em cuidados hospitalares agudos. Sendo grande parte associado ao cateter (Gould et al., 2009)

Segundo Agencia Nacional de Vigilância Sanitária (2017), qualquer infecção sintomática de trato urinário em pacientes de uso do cateter vesical de demora há pelo menos 48 horas, configura-se em infecção do trato urinário relacionado à assistência a saúde associada ao cateter vesical (ITU-AC).

Os indivíduos submetidos a qualquer tipo de instrumentação do trato urinário, como o cateterismo possuem um risco de infecção por via ascendente além de risco de infecção do trato urinário complicada. Sendo assim, o conhecimento de enfermagem a respeito desse critério da prática na assistência, a inserção de cateter vesical de demora e no controle de infecção hospitalar, deve ser construído desde a sua formação acadêmica. Para isso, necessita ter conhecimento cientifico teórico e técnico, pensar criticamente, analisar os problemas, encontrar soluções dentro dos princípios éticos e bioéticos da profissão a 
fim de que possa se tornar decisões rápidas e concretas, transmitindo segurança para sua equipe, paciente e familiar, diminuindo assim os riscos que ameaçam a vida do paciente (Merces et al., 2013)

Assim, esse estudo tem como questão norteadora: Qual o conhecimento sobre CVD dos alunos de graduação de enfermagem em uma instituição privada? Os objetivos desse estudo foram descrever o perfil do acadêmico de enfermagem de uma instituição privada e destacar o conhecimento dos alunos sobre Infecção do trato urinário (ITU) associado ao Cateterismo Vesical de Demora (CVD).

\section{Metodologia}

Trata-se de estudo descritivo de abordagem quantitativa. Este estudo foi realizado no segundo semestre de 2017 que visou à coleta de dados, de acadêmicos de enfermagem de todos os períodos do turno noturno através de um questionário estruturado e fechado elaborado pelos pesquisadores.

O campo de pesquisa foi uma instituição de ensino superior privada localizada em Niterói, Rio de Janeiro, Brasil. Essa instituição possui curso de bacharelado de enfermagem há 16 anos. No ano de 2017 essa instituição possuía 306 alunos matriculados.

Os critérios de elegibilidade foram: Inclusão: os alunos regularmente matriculados no segundo semestre de 2017, no curso de enfermagem, que aceitaram participar do estudo. Exclusão: alunos matriculados em disciplinas de mais de um período. A amostragem foi em sequência, sendo selecionados 156 participantes.

O questionário foi elaborado e estruturado em duas partes. Sendo a primeira parte baseado em explorar o perfil sociodemográfico do acadêmico e na segunda sobre o conhecimento a cerca do procedimento de CVD que foi construído baseado nos critérios de prevenção de infecção associado ao cateter urinário - 2009, do centro de controle de doenças e prevenção (CDC). Subdividido em quatro grupos de afirmações, sendo elas: Quanto ao critério de inserção (indicação) do Cateter de demora, Quanto à técnica de inserção, Quanto ao manejo, e Quanto à retirada, de maneira que os alunos assinalassem as que considerassem o correto.

No momento da coleta é esclarecido para todos os participantes que se trata de uma pesquisa voluntária, e que os dados coletados não será associados ao indivíduo uma vez que não será necessária a identificação do mesmo no questionário. Para serem incluídos na pesquisa, o participante deveria aceitar participar e assinar o Termo de Consentimento Livre e Esclarecido. O estudo refere-se a um projeto de pesquisa, de iniciação científica, submetido e aprovado no comitê de Ética em Pesquisa - CEP - da instituição de ensino privada sob o número CAAE 65105517.7.0000.5289, parecer 1986860, e atende a resolução 466/2012.

\section{Resultados}

Foram coletados 156 questionários no total de 306 alunos matriculados no curso, totalizando uma média de 49\% dos alunos que responderam o questionário e contribuíram para a pesquisa.

O primeiro período foi o que mais obteve questionários respondidos, atingindo $69 \%$ de alunos desse período. Vale lembrar que se trata da turma com maior número de discentes.

O sétimo período foi o que se obteve maior número de recusa em contribuir para a pesquisa, tornando o percentual de questionários respondidos desse período bem inferior aos demais, atingindo apenas $21 \%$ de discentes desse período.

Do total de 156 alunos participantes, observou-se a predominância do sexo feminino com 115 participantes (73,7\%), 37 do sexo masculino $(23,7 \%)$ e 4 dos participantes $(2,6 \%)$ não se declararam quanto ao gênero. A faixa etária dos participantes abrangeu jovens adultos de 18 anos á adultos acima de 41 anos.

A predominância foi entre os alunos de 18 a 23 anos com 33\% dos alunos entrevistados seguido por alunos com 24 a 
29 anos somando $19 \%$ desses. Não podendo deixar de ressaltar que alunos acima dos 30 anos mostra-se uma soma considerável uma vez que esse grupo totaliza uma média de $47 \%$ dos discentes participantes.

Quanto ao estado civil dos participantes a maioria se declara solteiro, sendo então 59\% dos alunos. Dentre os discentes participantes, que declaram ter filhos verificou-se que apenas 38\% possuem de 1 a 4 filhos. Desses alunos com filhos a predominância se deu aos indivíduos que tem pelo menos um filho com 18\%, e os que têm até 4 filhos representa apenas $1 \%$ do desse grupo.

A maioria dos estudantes exerce alguma atividade profissional, totalizando $72 \%$ dos entrevistados. Outros entrevistados declararam desemprego, aposentadoria, totalizando $1 \%$ do grupo. A maioria dos alunos tem uma jornada de trabalho maior que 40 horas semanais representando 32\% dos alunos, seguido de $19 \%$ dos que trabalham de 31 a 40 horas, $10 \%$ os que trabalham 21 a 30 horas, $7 \%$ representam os que trabalham sem jornada fixa ou em até 10 horas semanais, e a minoria trabalham de 11 a 20 horas semanais representando apenas $4 \%$ dos entrevistados

Em relação à atividade profissional, dividiu-se o grupo de participantes entre os que eram da área da saúde e os que exerciam outras atividades. Sendo classificados como da área da saúde apenas os alunos que se declararam técnicos ou auxiliares de enfermagem, e os demais entram no grupo como outros. Á partir de então, observou-se que esse grupo faz parte consideravelmente do nosso público, caracterizando cerca de $44 \%$ dos alunos participantes da pesquisa.

A renda dos entrevistados foi abordada de forma individual e não familiar. A predominância foi maior na renda de 1 a 3 salários mínimos (51\%), seguido dos que declararam não terem renda ou ser dependente dos pais (24\%), ter renda até 1 salário (18\%), de 3 a 6 salários (6\%) e de 6 a 10 salários (1\%). No questionário foi abordado também acima de 10 salários mínimos, porém não houve nenhum declarante para essa opção.

Com relação ao conhecimento dos alunos sobre CVD, as Tabelas 1, 2, 3 e 4 demonstra a média de pontos por período quanto ao critério de inserção, manejo e retirada da CVD. 
Tabela 1: Média de Acertos quanto ao Critério de Inserção do CVD.

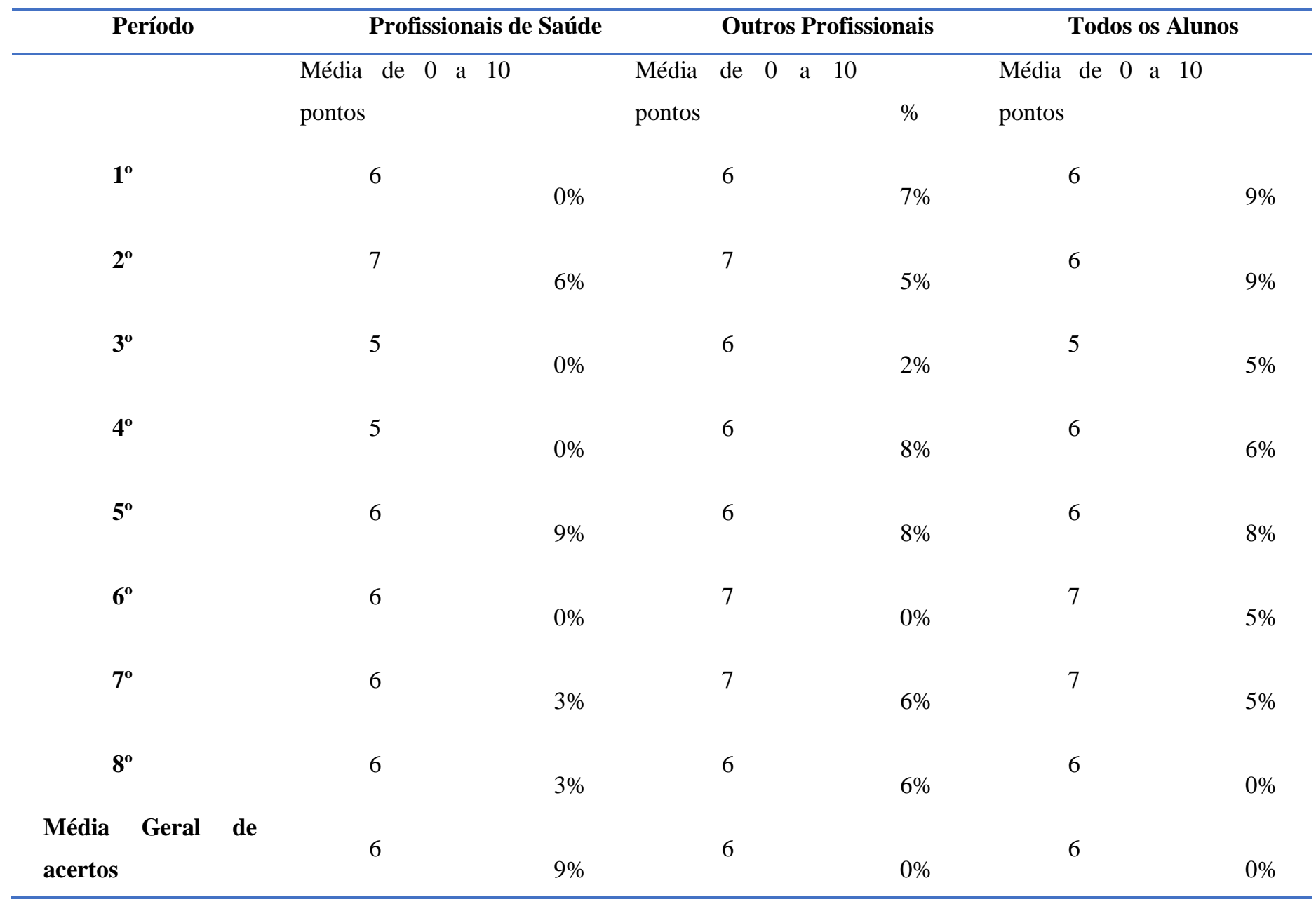

Fonte: Autores (2021).

Na Tabela 1 observa-se homogeneidade na média dos acertos, bem como nas tabelas 2, 3 e 4 quanto a outros aspectos relacionados a técnica de CVD foram identificados independente do período ou se o participante fosse profissional de saúde ou não. 
Research, Society and Development, v. 10, n. 3, e56210313784, 2021

(CC BY 4.0) | ISSN 2525-3409 | DOI: http://dx.doi.org/10.33448/rsd-v10i3.13784

Tabela 2: Média de Acertos Quanto ao Critério da Técnica de Inserção do CVD.

\begin{tabular}{|c|c|c|c|c|c|c|}
\hline \multirow{2}{*}{ íodo } & \multicolumn{2}{|c|}{ Profissionais de Saúde } & \multicolumn{2}{|c|}{ Outros Profissionais } & \multicolumn{2}{|c|}{ Todos os Alunos } \\
\hline & $\begin{array}{l}\text { Média de } 0 \text { a } \\
7 \text { pontos }\end{array}$ & $\%$ & $\begin{array}{l}\text { Média de } 0 \text { a } \\
7 \text { pontos }\end{array}$ & $\%$ & $\begin{array}{l}\text { Média de } \\
\text { pontos }\end{array}$ & $\%$ \\
\hline $1^{\circ}$ & 5 & $66 \%$ & 5 & $66 \%$ & 5 & $66 \%$ \\
\hline $2^{\circ}$ & 4 & $61 \%$ & 4 & $61 \%$ & 4 & $61 \%$ \\
\hline $3^{\mathbf{o}}$ & 5 & $71 \%$ & 5 & $71 \%$ & 5 & $71 \%$ \\
\hline $4^{\circ}$ & 5 & $64 \%$ & 4 & $61 \%$ & 4 & $61 \%$ \\
\hline $5^{\circ}$ & 5 & $66 \%$ & 4 & $63 \%$ & 5 & $64 \%$ \\
\hline $6^{0}$ & 5 & $69 \%$ & 5 & $64 \%$ & 5 & $66 \%$ \\
\hline $7^{\circ}$ & 4 & $61 \%$ & 3 & $47 \%$ & 4 & $54 \%$ \\
\hline $8^{\circ}$ & 5 & $69 \%$ & 5 & $66 \%$ & 5 & $67 \%$ \\
\hline $\begin{array}{l}\text { Média Geral } \\
\text { de acertos }\end{array}$ & 5 & $66 \%$ & 4 & $63 \%$ & 5 & $64 \%$ \\
\hline
\end{tabular}

Fonte: Autores (2021).

Tabela 3: Média de Acertos Quanto ao Manejo do CVD.

\begin{tabular}{|c|c|c|c|c|c|c|c|c|}
\hline \multicolumn{2}{|c|}{ Período } & \multicolumn{2}{|c|}{ Profissionais de Saúde } & \multicolumn{2}{|c|}{ Outros Profissionais } & \multicolumn{2}{|c|}{ Todos } & os \\
\hline & Média & & Médi & & Médi & & & \\
\hline & 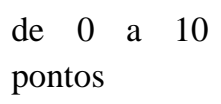 & $\%$ & $\begin{array}{l}\text { a de } 0 \text { a } 10 \\
\text { pontos }\end{array}$ & $\%$ & $\begin{array}{l}\text { a de } 0 \text { a } 10 \\
\text { pontos }\end{array}$ & $\%$ & & \\
\hline & $1^{\mathbf{o}}$ & 6 & $56 \%$ & 6 & $56 \%$ & 6 & $6 \%$ & \\
\hline & $2^{\circ}$ & 6 & $61 \%$ & 6 & $55 \%$ & 6 & $7 \%$ & 3 \\
\hline & $3^{\mathbf{o}}$ & 5 & $50 \%$ & 6 & 0,62 & 6 & $5 \%$ & \\
\hline & $4^{\circ}$ & 6 & $56 \%$ & 6 & $56 \%$ & 6 & $6 \%$ & \\
\hline & $5^{\circ}$ & 6 & $64 \%$ & 6 & $64 \%$ & 6 & $4 \%$ & C \\
\hline & $6^{\mathbf{0}}$ & 7 & $67 \%$ & 7 & $68 \%$ & 7 & $8 \%$ & $t$ \\
\hline & $7^{\circ}$ & 8 & $80 \%$ & 8 & $76 \%$ & 8 & $8 \%$ & \\
\hline & $8^{\circ}$ & 6 & $56 \%$ & 5 & $46 \%$ & 5 & $1 \%$ & \\
\hline $\begin{array}{l}\text { Média } \\
\text { Geral de } \\
\text { acertos }\end{array}$ & 6 & $61 \%$ & 6 & $60 \%$ & 6 & $61 \%$ & & \\
\hline
\end{tabular}

Fonte: Autores (2021). 
Tabela 4: Média de Acertos Quanto ao Critério de Retirada do CVD.

\begin{tabular}{|c|c|c|c|c|c|c|}
\hline Período & \multicolumn{2}{|c|}{ Profissionais de Saúde } & \multicolumn{2}{|c|}{ Outros Profissionais } & \multicolumn{2}{|c|}{ Todos os Alunos } \\
\hline & $\begin{array}{l}\text { Média de } 0 \\
\text { a } 4 \text { pontos }\end{array}$ & $\%$ & $\begin{array}{l}\text { Média de } 0 \\
\text { a } 4 \text { pontos }\end{array}$ & $\%$ & $\begin{array}{l}\text { Média de } 0 \\
\text { a } 4 \text { pontos }\end{array}$ & $\%$ \\
\hline $1^{\circ}$ & 3 & $63 \%$ & 2 & $58 \%$ & 2 & $60 \%$ \\
\hline $2^{\circ}$ & 3 & $75 \%$ & 2 & $60 \%$ & 3 & $65 \%$ \\
\hline $3^{\circ}$ & 1 & $28 \%$ & 3 & $80 \%$ & 2 & $50 \%$ \\
\hline $4^{\circ}$ & 3 & $63 \%$ & 2 & $48 \%$ & 2 & $50 \%$ \\
\hline $5^{\circ}$ & 3 & $75 \%$ & 3 & $68 \%$ & 3 & $70 \%$ \\
\hline $6^{\circ}$ & 3 & $70 \%$ & 2 & $45 \%$ & 2 & $58 \%$ \\
\hline $7^{\circ}$ & 3 & $83 \%$ & 2 & $50 \%$ & 3 & $65 \%$ \\
\hline $8^{\circ}$ & 3 & $65 \%$ & 2 & $58 \%$ & 3 & $63 \%$ \\
\hline Média & & & & & & \\
\hline $\begin{array}{l}\text { Geral de } \\
\text { acertos }\end{array}$ & 3 & $65 \%$ & 2 & $58 \%$ & 3 & $60 \%$ \\
\hline
\end{tabular}

Fonte: Autores (2021).

De uma forma geral, fazendo a análise do conhecimento do acadêmico a cerca do tema em todos os critérios os alunos do sexto e sétimo período se destacam dos demais com média de $66 \%$ e $67 \%$ dos acertos respectivamente. Porém observa-se uma distribuição homogênea nos acertos em todos os períodos (Figura 1).

Figura 1: Percentual Geral de Acertos dos Alunos no Conhecimento Sobre CVD.

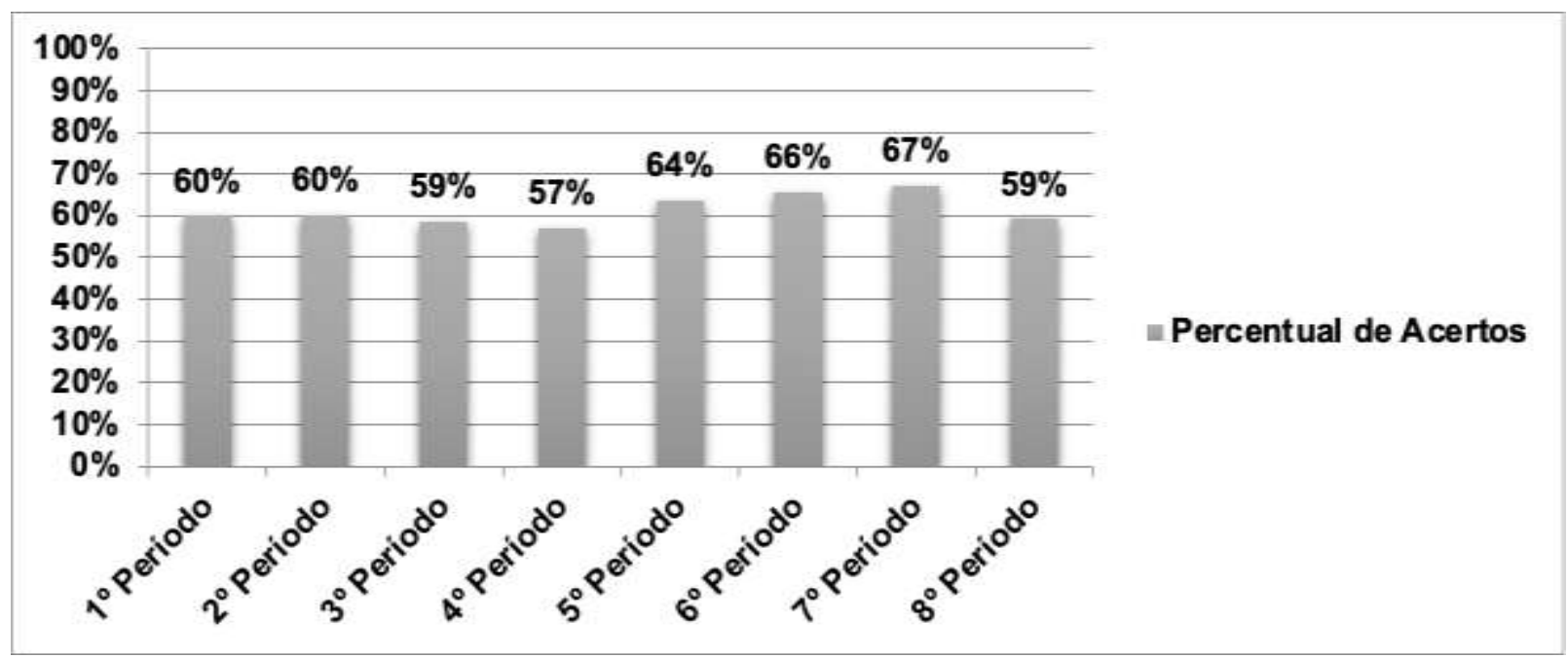

Fonte: Autores (2021).

\section{Discussão}

Nesse estudo verificou-se que nessa instituição privada alguns fatores sociodemograficos podem afetar o desemprenho acadêmico dos alunos. Começamos por constatar que o público maior é de alunos do sexo feminino (73,7\%). Sabe-se que a feminilidade da profissão não se encontra apenas nesse estudo, trata-se de uma característica nacional e mundial, 
embora haja crescente aumento do gênero masculino na enfermagem, esta permanece como eminentemente feminina, pois apesar do tempo decorrido desde o seu surgimento até os dias atuais, ainda se verifica um considerável percentual de mulheres que integra e busca a enfermagem como profissão, conforme evidenciam esses números (Brasil, 2013; Souza et al., 2013). Sendo a ideia de cuidar, de proteger, faz parte da história da mulher e com ela, ficaram as obrigações domésticas, o cuidado, a educação dos filhos, a assistência aos idosos e enfermos da família. Por extensão, o cuidado ao doente também passou a ser trabalho feminino, uma vez que exigia tarefas similares (Souza et al., 2013).

Nesta pesquisa temos o público maior na faixa etária entre 18 a 30 anos (52\%), levando em consideração que $47 \%$ representam a faixa etária dos 31 até 41 anos. Apesar dos alunos em sua maioria serem de uma população jovem, não se pode desconsiderar os poucos adultos de meia idade que muitas vezes possuem as suas dificuldades no uso das tecnologias facilitadoras do aprendizado bem como o cansaço físico e mental, que dificultam o processo de aprendizagem.

A maioria dos alunos é solteira (59\%), e não possui filhos (62\%) sendo um número considerável de casados com $29 \%$ e $38 \%$ com pelo menos um filho. Portanto a associação da vida acadêmica com a doméstica pode ser um dificultador do processo de aprendizagem desses discentes pela condição de acumulo de funções.

Também são consideradas dificuldades no período de formação: a falta de tempo para o lazer, a família, os amigos e as necessidades pessoais. Assim, identifica-se que o graduando de enfermagem, depara-se diariamente com situações que exigem adaptações (Bublitz et al., 2012)

Também é caracterizado como um público trabalhador, sendo que $72 \%$ dos entrevistados trabalham. Nos últimos anos, tem se percebido uma mudança no perfil dos ingressantes da graduação em Enfermagem. Tal perfil constitui-se por alunos já inseridos no mercado de trabalho, seja na área de saúde ou não (Melo et al., 2016)

Os trabalhadores buscam a graduação não só motivados pelo saber, mas também pelo desejo de melhores condições de trabalho e salários, pois, na medida em que evoluem na construção do conhecimento, têm perspectivas de mudança do padrão financeiro, o que demonstra coragem e determinação na busca de seus objetivos, considerando-se que é árdua a batalha de estudar e trabalhar (Fontana; Brigo, 2012)

Em sua maioria (51\%) relatam ganhar de um a três salários mínimos. Em outro estudo de realizado em 2013 com estudantes de enfermagem na Universidade Estadual do Rio de Janeiro, revela um público semelhante quanto à renda familiar, refere que 52,69\% dos ingressantes possuíam uma renda familiar de até três salários mínimos, que correspondia à $\mathrm{R} \$ 1.395,00$, onde relata que está renda mostra-se desfavorável para fazer frente aos gastos relativos à permanência de um estudante no ensino superior, pois há despesas com transporte, alimentação, material didático e vestuário (Souza et al., 2013)

Nos resultados a cerca do conhecimento acadêmico sobre CVD, não houve um período que se destacasse dos demais. O percentual de aproveitamento dos alunos no geral variou-se de $57 \%$ a $67 \%$. Sendo o sexto período e o sétimo com o melhor aproveitamento $66 \%$ e $67 \%$ respectivamente. Supõe-se que esse resultado homogêneo, ocorre por vários dificultadores do processo da pesquisa, dentre eles: os profissionais de saúde que se integram em todos os períodos da graduação de enfermagem, a elevada carga horária de trabalho, a falta de tempo para as atividades extracurriculares para associar a teoria à prática.

Quando separado os grupos que obtiveram um melhor aproveitamento com os de menor aproveitamento observa-se que os alunos que obtiveram mais de 70\% de aproveitamento, em sua maioria são jovens com menos de 30 anos, solteiros, não possuem filhos, moram no mesmo município da universidade (Niterói), trabalham e tem sua jornada maior que 40 horas semanais, são profissionais da área da saúde. No grupo que obtiveram aproveitamento inferior a $45 \%$, em sua maioria são também solteiros, não possuem filhos, diferentemente do grupo anterior o município de moradia desses não é o mesmo da universidade tendo então um período de deslocamento maior, a jornada de trabalho é superior a 30 horas semanais e são de outras áreas profissionais. Sugere-se então que apesar da alta jornada de trabalho a pratica do profissional de enfermagem 
possibilita-o ao melhor aproveitamento a cerca do tema uma vez que o mesmo tem a oportunidade de vivenciá-lo em seu local de trabalho, diferente dos outros que obtiveram um menor aproveitamento que além de não estar inserida no âmbito da assistência a saúde o mesmo ainda tem outros dificultadores em seu processo de aprendizagem como, por exemplo, o deslocamento maior para a universidade.

A Formação Profissional, entendida como a preocupação com o conhecimento adquirido na fase de formação acadêmica e o seu impacto na vida profissional também é percebida como estressante por estudantes de enfermagem de contextos socioculturais diferentes (Benavente et al., 2014).

O procedimento de cateterização é complexo e invasivo, além de trazer consequências para o paciente tais como trauma de uretra ou da bexiga pela inserção incorreta, e introdução de microrganismos no trato urinário que podem resultar em infecção (Melo et al., 2016)

Dessa forma, o procedimento exige o conhecimento, a adesão estrita à técnica asséptica, e a habilidade técnica, tornando-se importante compreender o panorama de conhecimento dos graduandos para que possamos atuar ainda na base para aprimorar a formação de nível superior da enfermagem.

\section{Conclusão}

A partir das dúvidas que motivaram a pesquisa, percebe-se que o perfil do acadêmico de enfermagem que ingressa em uma universidade privada, trata-se de um público de adultos jovens que já estão inseridos no mercado de trabalho e buscam a universidade, motivados pela ascensão da carreira, bem como melhoria de salários.

Quanto ao conhecimento do acadêmico de enfermagem sobre ITU-AC observa-se que na universidade o conhecimento dos acadêmicos torna-se homogêneo quando comparado no geral, mas quando separado dos profissionais de saúde, estes possuem um número de acertos maior, sugerindo que a atividade profissional pode ser um facilitador para assimilação do conteúdo.

Pode-se sugerir, então, que o perfil sociodemográfico dos alunos de enfermagem não interferiu no processo de aprendizagem e assimilação do conteúdo. A partir da identificação dessas fragilidades, pode-se atuar em alguns pontos deficitários do acadêmico como na inserção e incentivo do mesmo à atividades de extensão, melhoria na qualidade das aulas práticas tornando facilitadores do processo de assimilação dos conteúdos.

Diante desses resultados, sugere-se outras pesquisas relacionadas aos dificultadores do processo de aprendizagem e o conhecimento do aluno sobre ITU-AC. Bem como uma comparação do perfil acadêmico e o conhecimento do mesmo a cerca do tema em universidades públicas como comparativo e objetivo de melhoria na qualidade de educação e formação dos profissionais de enfermagem.

\section{Referências}

Benavente, S. B. T., Silva, R. M., Higashi, A. B., Guido, L. A., \& Costa, A. L. S. (2014). Influência de fatores de estresse e características sociodemográficas na qualidade do sono de estudantes de enfermagem. Rev. Esc. Enferm. USP. 38(3): 514-520.

Brasil. Agência Nacional de Vigilância Sanitária (2017). Medidas de Prevenção de Infecção Relacionadas à Assistência à Saúde. Brasília: ANVISA.

Brasil. (2013). Pesquisa Perfil da enfermagem no Brasil - Banco de dados. Brasília: COFEN.

Brasil. (2015). Procedimento de sondagem vesical não pode ser delegado confirma resolução 450/2013 sobre o procedimento de al ta complexidade. Conselho Federal de Enfermagem: Brasília. http://www.cofen.gov.br.

Bublitz, S., Freitas, E. O., Kirchhof, R. S., Lopes, L. F. D., \& Guido, L. A. (2012). Estressores Entre Acadêmicos De Enfermagem De Uma Universidade Pública. Rev. Enfer. UERJ. 20(6): 739-45.

das Merces, M. C., Carvalho, M. A. M., Araújo, P. R. S, Queiroz, A. B. Q., Silva, B. S. M., \& Sousa, M. N. M., \& Servo, M. L. S. (2013). A prática do(a) enfermeiro(a) na inserção do cateter de Folley em pacientes de unidade de terapia intensiva: limites e possibilidades. Rev. Epidemiologia e Controle de infecção. 3(2): 55-61. http://dx.doi.org/10.17058/reci.v3i2.3157 
Research, Society and Development, v. 10, n. 3, e56210313784, 2021

(CC BY 4.0) | ISSN 2525-3409 | DOI: http://dx.doi.org/10.33448/rsd-v10i3.13784

Ercole, F. F., Macieira, T. G. R., Wenceslau, L. C. C., Martins, A. R., Campos, C. C., \& Chianca, T. C. M. (2013). Revisão integrativa: Evidencias na prática do cateterismo urinário intermitente/demora. Rev. Latino-Am. Enfermagem. 21(1),. http://dx.doi.org/10.1590/S0104-11692013000100023.

Fontana, R. T., \& Brigo, E. L. (2012). Estudar e Trabalhar: Percepções de Técnicos de Enfermagem Sobre Esta Escolha. Escola Anna Nery. 16(1): 128-133. https://doi.org/10.1590/S1414-81452012000100017.

Gould, C. V., Umscheid, C. A., Agarwal, R. K., Kuntz, G., \& Pegues, D. A. (2009). Guideline For Prevention Of Catheter associated Urinary Tract Infections 2009. Centers for Disease Control and Prevention - CDC

Melo, G. S. M., Tibúrcio, M. P., Freitas, C. C. S., Vasconcelos, Q. L. D. A. Q., Costa, I. K. F., \& Torres, G. V. (2016). Semiologia e semiotécnica da enfermagem: avaliação dos conhecimentos de graduandos sobre procedimentos. Revista Brasileira de Enfermagem. 70(2): 249-256. https://doi.org/10.1590/0034-7167-2016-0417

Pereira, A. S., Shitsuka, D. M., Pereira, F. J. \& Scitsuka, R. (2018). Metodologia da pesquisa científica. UFSM

Souza, N. V. D. O., Penna, L. H. G. P., Cunha, L.S., Baptista, A. A. S., Mafra, I. F., \& Mariano, D. C. A. (2013). Perfil socioeconômico e cultural do estudante ingressante no curso de graduação em enfermagem. Rev. Enferm. UERJ. 21(6): 718-722. 